\title{
Évolution et perspectives du management, de l'Antiquité à la Renaissance : à la recherche des leçons perdues
}

Sous la direction de Georges Nurdin, Paris, L'Harmattan, coll. un autre regard, 2012, 137 p., 15, $50 €$

\section{Annick Schott}

\section{(2) OpenEdition}

\section{Journals}

Édition électronique

URL : http://journals.openedition.org/communicationorganisation/3819

DOI : 10.4000/communicationorganisation.3819

ISSN : 1775-3546

Éditeur

Presses universitaires de Bordeaux

Édition imprimée

Date de publication : 1 juin 2012

Pagination : 213-215

ISBN : 978-2-86781-827-9

ISSN : $1168-5549$

\section{Référence électronique}

Annick Schott, «Évolution et perspectives du management, de l'Antiquité à la Renaissance : à la

recherche des leçons perdues », Communication et organisation [En ligne], 41 | 2012, mis en ligne le 29 mars 2013, consulté le 22 septembre 2020. URL : http://journals.openedition.org/

communicationorganisation/3819; DOI : https://doi.org/10.4000/communicationorganisation.3819 


\section{Évolution et perspectives du management, de l'Antiquité à la Renaissance : à la recherche des leçons perdues, sous la direction de Georges NURDIN, Paris, L'Harmattan, coll. un autre regard, 2012, 137 p., 15, $50 €$}

Le projet éditorial de cet ouvrage est de démontrer grâce à la réunion d'auteurs de disciplines très diverses (archéologie, économie, finances, histoire, lettres, philosophie) que le management n'est pas une invention moderne et qu'il n'est pas non plus universel. Un clin d'œil est fait au management dans un contexte multiculturel qui nécessite, à partir des enseignements de l'empire romain, une prise de conscience et une connaissance des différenciations culturelles.

Cinq articles se succèdent, avec pour chacun d'entre eux des thématiques différentes, mais qui convergent.

Le management stratégique est une question fondamentale, de lui dépend, plus encore en période de crise, la survie et le développement de l'entreprise.

Lidée qui émerge des oppositions " actuelles " entre gouvernance et réduction de coût ; contrôle et autonomie, est que la vision mécaniste de l'entreprise a gagné avec en plus une obsolescence programmée des biens, faisant du consommateur un acteur majeur de la croissance!

Les pères fondateurs de la pensée managériale sont aussi ceux qui ont vécu aux environ de 4500 av. J.C. Non seulement les documents comptables et les méthodes utilisées (comptabilité d'engagement et comptabilité en partie double) en attestent, mais les informations traitées étaient destinées aux parties prenantes (actionnaires, propriétaires, managers). Ainsi la preuve est apportée que la plupart des méthodes et des modes de gestion utilisés aujourd'hui datent de l'Antiquité.

Mieux, les approches managériales ont toutes une dette envers Aristote. Il est vrai que ce dernier, notamment dans son traité « Les Economiques » accorde un statut particulier à la capacité de penser les réalités à égale distance entre rigueur mathématique et empirisme complet. A partir d'extraits, sont réfléchis les enjeux de cet engouement pour l'éthique aristotélicienne appliquée à l'économie contemporaine.

La place de l'économie est circonscrite dans la pensée grecque. En grec le mot " oiconomia » signifie gestion régulée d'une maison. Ainsi, l'économie est au service de la réalité supérieure qu'est la vie en collectivité ou la politique. Cependant l'aléatoire où se situe l'économie lui permet de réinventer des 
espaces de liberté. Mais celle-ci, quel que soit son champ d'application, doit rester «dans la juste mesure ». Est posée la question du respect des limites et du sens de la mesure qui renvoie à l'éthique et au bien vivre plutôt que d'orienter son appétit de vivre sans limite : "l'individuelle âpreté du gain devient folie corruptrice pour la collectivité ».

Pour penser l'économie, Aristote décrit et analyse le statut du "prudent " et du valeureux ", qui permet à la figure managériale contemporaine de se positionner. Ce statut sait combiner chance et risque de l'action humaine (moment opportun, juste mesure, évaluation précise entre risques et bénéfices). Ainsi le manager «ne peut agir dans le chaos de l'empirisme, en tant qu'homme d'action qui gère des affaires économiques; il ne peut se contenter d'agir au cas par cas ; " il a aussi besoin de conceptualiser le réel. C'est dans cette navigation entre deux mondes que se situe la sagesse pratique d'Aristote. Celle-ci est bien évidemment empreinte d'une éthique qui donne des valeurs. L'exemplaire fonde ses bonnes pratiques sur des valeurs qu'il détermine. Cette conception du management est intéressante aujourd'hui puisqu'elle tient compte des Hommes tels qu'ils sont et non tels qu'ils devraient être.

Puisque c'est au dirigeant de construire son éthique personnelle à partir des valeurs collectives qu'il choisira, il y a peu de chance pour que ses bonnes pratiques entrent dans un formatage et une standardisation. En somme, toutes les bonnes pratiques ne se valent pas, et toute éthique dans l'entreprise n'est pas que subjective. Cependant, selon Aristote, "seule une décision politique peut harmoniser les décisions auxquelles l'économie privée est nécessairement soumise. »

D'autres visions managériales viennent compléter celle-ci. Ainsi celle de Sum Tzu contenue dans son ouvrage «l'art de la guerre » rédigé au $5^{\circ}$ siècle av. J.C. et traduit en France depuis 1772. Dans la pensée traditionnelle chinoise, trois facteurs peuvent amener à la victoire. Dans leur traduction managériale ces trois facteurs sont la connaissance de l'environnement (économique, politique, sociologique, etc.), l'avantage terrain (richesses locales autant matérielles qu'humaines) et enfin l'entente des hommes sur notamment la compréhension et la réalisation des objectifs. Des indications sur les attributs du leader sont toujours d'actualité : il lui faut donner du sens et la voie à suivre ; être dans une sagesse pratique, telle que définie par Aristote ; dans une recherche d'équité tant dans la récompense que la sanction, système qui repose sur le couple responsabilité / exemplarité ; la bienveillance ; le courage (prise de risques) et la rigueur (application de la discipline). La discipline renvoie à la somme des moyens employés pour diviser le travail en tâches et s'assurer de la coordination entre les tâches.

Sur le plan tactique quatre leviers permettent de conduire une armée ou une entreprise. S'adapter aux variations de l'environnement exogène comme endogène (se connaître et connaître son environnement). Considérer les 
points faibles et les points forts (pour se concentrer sur les marchés les moins saturés (analyse des forces et faiblesses de l'entreprise / des menaces et opportunités des concurrents). Etre porté par la force de l'élan, c'est créer de l'élan en générant une situation ou un environnement favorable en accumulant de l'énergie, en prenant des initiatives et en agissant avec rapidité. En d'autres termes, l'entreprise doit améliorer son environnement interne en tenant compte des ressources dont elle dispose. Elle doit ensuite prendre des initiatives (commerciales, technologiques, etc....). Enfin elle doit être rapide sans se hâter dans un monde incertain, afin de saisir des opportunités. Utiliser des informateurs (espions), cette pratique s'est développée au regard de la dureté commerciale et de la nécessité d'accéder à des informations sur l'adversaire.

En terme de management, le traité « l'art de la guerre » révèle des paradoxes : adaptation aux événements // création de l'élan; structure rigide et discipline stricte // flexibilité et création.

Plus contemporaines, des figures de l'apogée culturelle et intellectuelle de l'Islam du $\mathrm{XI}^{\circ}$ siècle vont marquer l'évolution des concepts économiques et managériaux ; par exemple en insistant sur la défense de l'égalité ou de justice sociale dans la relation de travail et dans la redistribution des richesses. Ces idées ont été reprises avant la Renaissance en occident par des savants et des praticiens religieux ou pas. Sont ainsi posés les prémisses d'un management collaboratif enrichissant patrons et compagnons.

Si l'objet de cet ouvrage collectif est de pointer du doigt que de manager des hommes, qui plus est de la bonne manière, est vieux comme le Monde, il est de rappeler que le management repose sur la capacité à être visionnaire et donc à connaitre les richesses globales de l'entreprise et les caractéristiques de son environnement.

Annick Schott

\section{Benoit CORDELIER, Changement organisationnel et management par projet : mobilisation des systèmes d'information, Paris, L'Harmattan, 2012, 25 p. , 50€}

Benoît Cordelier, professeur au département de communication sociale et publique de l'Université du Québec à Montréal (UQAM, Canada) fait paraître une version remaniée de sa thèse intitulée ici : «Changement organisationnel et management par projet. Mobilisation des systèmes d'information ». Nous reconnaîtrons, en effet, l'exigence épistémologique et méthodologique qui structure le propos et qui donne toute sa richesse à l'analyse de terrain.

Immergé au sein d'une entreprise de spiritueux dans le cadre d'une observation participante, Benoît Cordelier nous donne à voir l'évolution 